\title{
BER Analysis with Adaptive Modulation Coding in MIMO-OFDM for WiMAX using GNU Radio
}

\author{
B. Siva Kumar Reddy ${ }^{\mathrm{a}^{*}}$ and B. Lakshmi ${ }^{\mathrm{b}}$ \\ ${ }^{a}$ Research Scholar and Associate Professor Department of Electronics and Communication Engineering \\ National Institute of Technology, Warangal Andhra Pradesh, India-506004
}

\begin{abstract}
This paper explores how MIMO increases channel capacity and how the fundamental characteristics of a MIMO system can create significant test challenges. Spatial modulation (SM) is the latest developed modulation method in the field of communication. The latest research on this shows that SM is useful to achieve the multiplexing gain for the single antenna system, this avoids the inter-channel interference but the spatial modulation is inherently unable to get transmit-diversity. In other words, SM is a novel modulation technique which combines the high multiplexing gain provided by the spatial modulation and transmit-diversity gain, given by the space time block codes (STBCs) technology. Different space-time block coding (STBC) schemes including Alamouti's STBC for 2 transmit antennas as well as orthogonal STBC (OSTBC) for 3 and 4 transmit antennas are explored. The result of using these MIMO techniques is higher data rate or longer transmit range without requiring additional bandwidth or transmit power. This paper presents a detailed study of diversity coding for MIMO systems. In addition, adaptive modulation and coding (AMC) technique in conjunction with MIMO techniques constitute a technological breakthrough that greatly helps in satisfying the ever increasing demands of wireless networks. This paper presents a performance study of Mobile WiMAX networks based on MIMO and AMC perspectives. It also describes how to implement WiMAX PHY with MIMO on software defined radio (SDR) experimental setup with the help of USRP N210 as hardware and GNU Radio as software platforms.
\end{abstract}

Index Terms: AMC, GNU Radio, MIMO-OFDM, STBC, OSTBC, USRP N210, WiMAX.

(C) 2014 Published by MECS Publisher. Selection and/or peer review under responsibility of the Research Association of Modern Education and Computer Science

\section{Introduction}

Mobile WiMAX [1] is developed to provide broadband access anywhere, anytime and on virtually any device at high vehicular speeds, with excellent quality of service (QoS). Mobile WiMAX introduces the.

* Corresponding author.

E-mail address: bsivakumar100@gmail.com 
concept of scalable OFDMA [2] using flexible fast Fourier transform (FFT) sizes and channel bandwidths

The FFT sizes and channel bandwidths vary from 128 to 2048 and 1.25 to $20 \mathrm{MHz}$, respectively. This scalable physical layer allows operators to deploy networks in different regions based on regulatory and market conditions.

In wireless communication, there is a demand not only for voice and data services, but also for multimedia services. WiMAX standard supports a full-range of smart antenna techniques, including spatial transmit diversity and spatial multiplexing (SM) [3]. Spatial transmit diversity is achieved by applying Alamouti's space time coding [4]. SM can also be employed to increase the error-free peak throughput. Higher order modulation schemes with SM increase the link throughput, but require high SNR to achieve low packet error rates (PER). Space time block coding (STBC) provides strong diversity gain, but cannot increase the link throughput without the use of Adaptive Modulation and Coding (AMC) [5], and therefore AMC has become a standard approach in recently developed wireless standards, including WiMAX. The idea behind AMC is to dynamically adapt the modulation and coding scheme to the channel conditions so as to achieve the highest spectral efficiency at all times. Adaptive modulation changes the coding scheme and/or modulation method depending on channel-state information - choosing it in such a way that it squeezes the most out of what the channel can transmit. Transmit diversity scheme employing space time block code (STBC) helps to increase number of antennas. This scheme has been presented by Alamouti [4]. He also showed the possibility of implementing such a scheme for a 2x2 MIMO system and pointed to a generalization of 2xM MIMO system, where M is the number of receiving antennas. This scheme has been modified by Tarokh et. al. [6], [7] to increase order of diversity in Multiple Input Multiple Output (MIMO). The overall effects of multiple inputs multiple output system can be summarized in terms of reduction of the bit error rate increase in system capacity and more efficient use of the transmitted power.

A software defined radio (SDR) [8], is a radio communication framework where the hardware components (e.g. mixers, amplifiers, filters, detectors, modulators/demodulators, and so on.) executed by method of programming on a personal computer. SDR utilizes USRP [9] as a hardware stage and GNU Radio [10] as a software. The execution of data transmission over wireless channels is well captured by watching their BER, which is a capacity of SNR at the receiver. The WiMAX PHY layer is executed utilizing different coders (Convolutional Coder, RMG (Reed-Muller-Golay) coder and RM (Reed-Muller) coder) in GNU Radio. In wireless channels, different models have been prescribed and utilized to calculate SNR. All the models utilization of the separation between the sender and the receiver, the channel gain, the path loss exponent. A few probability distributed functions are utilized to model a time-variant parameter i.e. channel gain. This paper portrays two frequently used distributions i.e. AWGN and Rayleigh models [11].

The rest of paper is organized as follows. The experimental setup which is used to implement WiMAX PHY is demonstrated in Section 2. MIMO-OFDM system model is described well with required equations in Section 3. Space diversity techniques such as spatial multiplexing (SM), space time block coding (STBC) and orthogonal space time block coding (OSTBC) techniques presented separately in Section 4. Adaptive modulation and coding technique is described in Section 5. The techniques presented in Section 4 and Section 5 are integrated with WiMAX PHY and demonstrated in Section 6. The conclusions drawn from Section 6 are depicted in Section 7.

\section{Software Defined Radio Testbed}

SDR platform solves incompatible wireless network issues by implementing radio functionalities as software modules running on generic hardware platforms. The radio functionalities include the modulation format and coding techniques. These radio functionalities can be changed without changing the hardware.

SDR comprises of RF section, IF section and baseband processing section. RF and IF sections are incorporated in USRP and baseband processing is performed in a PC using GNU radio companion (shown in Fig. 1). The USRP N210 allows for high-bandwidth, high-dynamic range processing capability. This includes a Xilinx® Spartan® 3A-DSP 3400 FPGA (field programmable gate array), two $100 \mathrm{MS} / \mathrm{s}$ ADCs, two $400 \mathrm{MS} / \mathrm{s}$ 
DACs and Gigabyte Ethernet connectivity to flow information to and from host processors. All baseband signal processing (e.g. modulation, amplification, mixing, filtering etc.) is done in GNU Radio. GNU Radio is a free software development toolkit that offers the signal processing runtime and readily available more than 100 processing blocks to implement software radios employing low-cost external RF hardware (USRP) and allows real time SDR applications [12]. In GNU Radio, signal processing blocks are written in Python and those are connected using $\mathrm{C}++$ and these both languages are communicated by SWIG (simplified wrapper and interface generator) interface compiler. Thus, the developer is allowed to accomplish real-time, high-throughput radio systems in a simple to-use, rapid-application development environment. In this paper all GNU Schematics (Signal flow graphs) are drawn for Mobile WiMAX specifications (FFT size=1024).

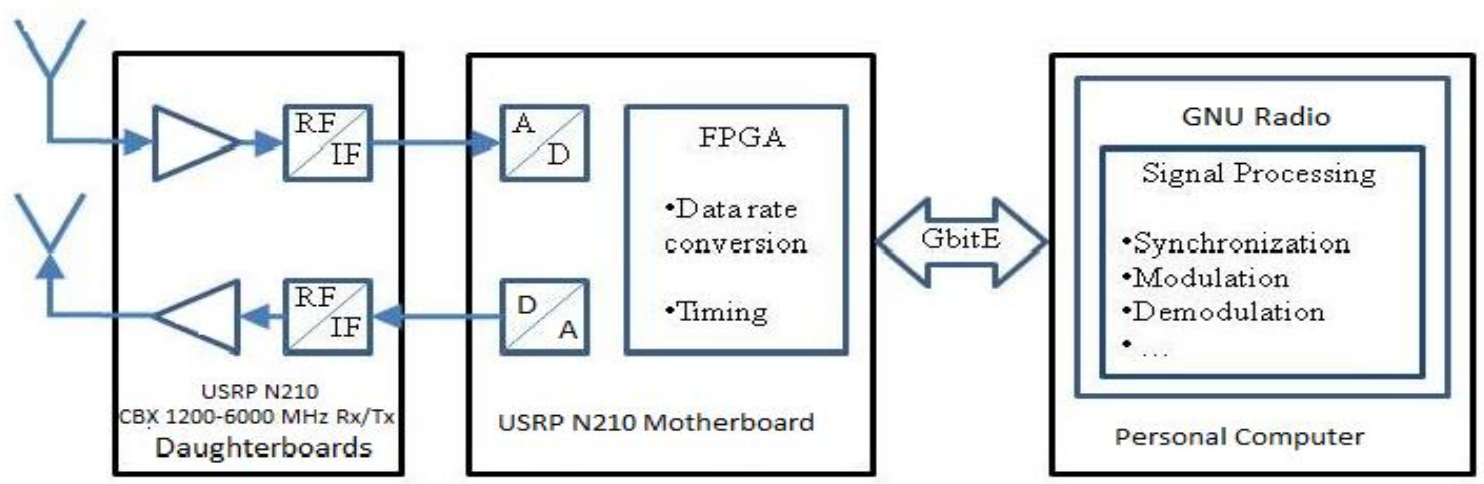

Fig. 1. Software defined radio block diagram with USRP N210 and GNU Radio.

\subsection{Benefits of SDR platform}

1. SDR is a reusable platform as all the features are implemented in software.

2. The implementation of additional features on SDR platform is cheaper. The cross over of SDR is also lower.

3. The architecture is highly flexible.

4. Flexibility is the key feature of the SDR implementation.

5. SDR being a reconfigurable architecture has low power consumption.

6. Lead time of SDR is also short. Lead time is the time taken between the initial stage of the system and appearance of results.

The signal transmitted from the transmitter section of SDR is in the form of digital pulses. In high frequency transmission, as the time period of the signal varies inversely with the frequency, the width of the pulse is chosen to be small.

\section{MIMO-OFDM System Model}

Multiple input multiple output uses multiple antennas at both sides which provides transmit diversity and receiver diversity. It's applicable in every kind of networks like PAN, LAN, WLAN, WAN, MAN. MIMO system can be applied in different ways to receive either a diversity gain, capacity gain or to overcome signal fading. MIMO system consists of three components, mainly transmitter, channel and receiver. Transmitter sends a multiple data such as $x_{1}, x_{2}, x_{3} \ldots x_{N}$ say $x_{i}$ from different transmit antenna and signal is received by each receive antenna $\left(r_{1}, r_{2}, r_{3} \ldots . r_{N}\right.$ say $\left.r_{j}\right)$ simultaneously. The relation between transmit data and receive data is given by [13] 


$$
\begin{aligned}
& r_{1}=h_{11} x_{1}+h_{12} x_{2}+\ldots \ldots \ldots \ldots+h_{1 N} x_{N} \\
& r_{2}=h_{21} x_{1}+h_{22} x_{2}+\ldots \ldots \ldots \ldots+h_{2 N} x_{N} \\
& \cdots \cdots \\
& r_{N}=h_{N 1} x_{1}+h_{N 2} x_{2}+\ldots \ldots \ldots+h_{N N} x_{N}
\end{aligned}
$$

The MIMO signal model is described as

$$
r=H x+n
$$

where $\mathrm{r}$ is $\mathrm{N}_{\mathrm{r}} \times 1$ received signal vector, $H$ is $N_{r} \times N_{t}$ the channel matrix, $x$ is $N_{t} \times 1$ transmitted vector and $n$ is $N_{r} \times 1$ Gaussian noise vector.

With $N_{t}$ inputs and $N_{r}$ outputs the channel can be expressed as $N_{r} \times N_{t}$ channel matrix $H$. By showing the channel in a matrix form, we can fully recover the transmitted data. The channel matrix can be represented as:

$$
H=\left[\begin{array}{cccc}
h_{11} & h_{12} & \ldots & h_{1 N_{t}} \\
h_{21} & h_{22} & \ldots & h_{2 N_{t}} \\
\cdot & \cdot & \cdot & \cdot \\
\cdot & \cdot & \cdot & \cdot \\
h_{N_{r} 1} & h_{N_{r} 2} & \ldots & h_{N_{r} N_{t}}
\end{array}\right]
$$

where $h_{i j}$ is the attenuation and phase shift between the $j$ th transmitter and $i$ th receiver. It is assumed the MIMO channel behaves in the quasi static manner.

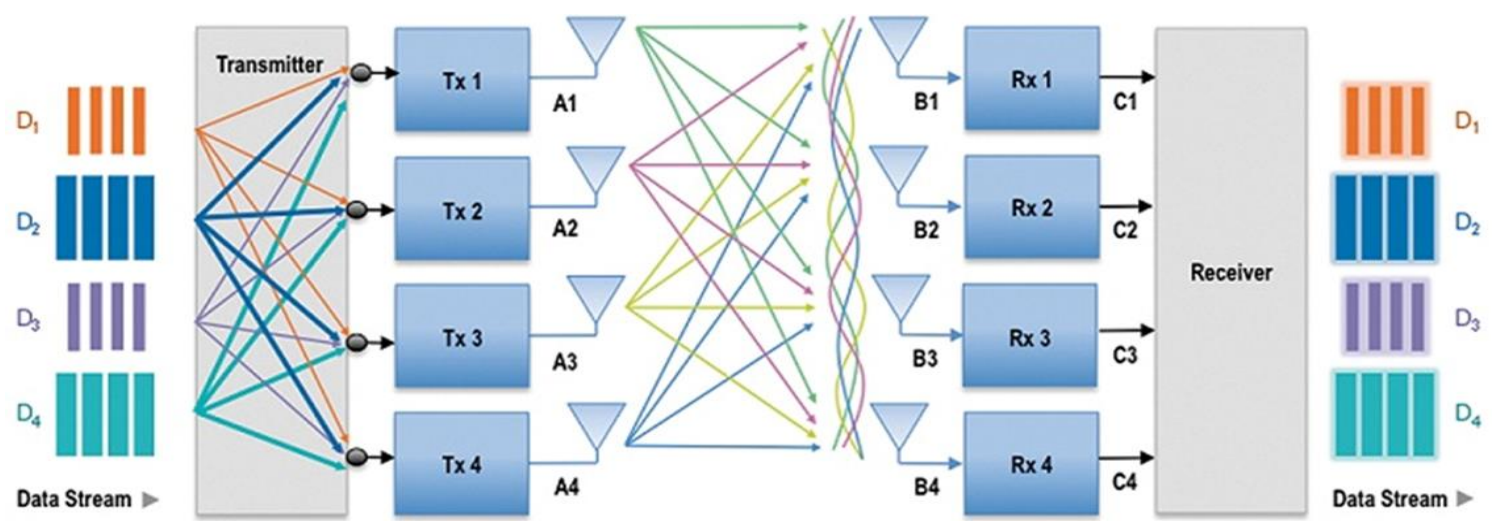

Fig. 2. Block diagram of multi input multi output (MIMO) system.

\section{Space Time Processing Techniques}

Space time processing technique for MIMO generally has two objectives one is to increase the data rate and next is to achieve maximum possible diversity. The space time processing techniques are: 


\subsection{Spatial Multiplexing}

Spatial multiplexing is a transmission technique to transmit several different data bits called streams through an independent spatial channel to achieve the greater throughput. Typically there are four kinds of spatial multiplexing schemes V-BLAST, diagonal blast, horizontal blast and turbo blast. Among them V-BLAST is the most promising scheme to apply.

The input bits stream is divided into $\mathrm{N}$ independent substreams using serial to parallel demultiplexer, and each stream is transmitted from several different antennas with output $\mathrm{N}$ symbol per channel. So the throughput increases $\mathrm{N}$ times and therefore, spatial multiplexing becomes the better candidate for high data rate [3].

\subsection{Space Time Coding}

To gain the maximum capacity of MIMO wireless channel one of the efficient procedures is to utilize space time coding. In STC, the multiple copies of information are transmitted for achieving diversity is extracted from a space time encoder which encodes a single bit through space and time. So coding is done in both spatial and temporal axis to correlate the transmitted signal from various transmit antenna at a different time. STC can achieve transmit diversity and power gain without losing the bandwidth. The space time coded matrix is given by

$$
\left[\begin{array}{cc}
x_{1} & x_{2} \\
-x_{2}^{*} & x_{1}^{*}
\end{array}\right]
$$

\subsection{Space Time Block Code}

Space Time Block Code (STBC) is based on the theory of orthogonal design. Using this theory space time block code can be constructed for any kind of transmit antenna. STBC can achieve full transmit diversity allowing maximum likelihood decoding algorithm based only on linear processing at the receiver [13]. STBC is constructed by $N_{t} * p$ transmission matrix $X$ where $N_{t}$ represents the number of transmit antenna and $\mathrm{p}$ represents the number of transmission period to transmit coded symbol through transmit antenna. Let $\mathrm{k}$ be the input number of symbol to an encoder in each encoding operation. So the rate of Space time block code is a ratio between the number of input symbols and number of space time coded symbols. Rate of STBC is [14]

$$
R=k / p
$$

Orthogonal design: An orthogonal design of size $N_{t} \times N_{t}$ with transmission matrix $X_{n}$ exists if and only if a number of transmit antennas are $N_{t}=2$ or 4 . Examples of orthogonal design are

When $N_{t}=2$

$$
\left[\begin{array}{ll}
x_{1} & -x_{2} \\
x_{2} & -x_{1}
\end{array}\right]
$$

When $N_{t}=4$ 


$$
\left[\begin{array}{cccc}
x_{1} & -x_{2} & -x_{3} & -x_{4} \\
x_{2} & x_{1} & x_{4} & -x_{3} \\
x_{3} & -x_{4} & x_{1} & x_{2} \\
x_{4} & x_{3} & -x_{2} & x_{1}
\end{array}\right]
$$

From the above matrix, it can be analyzed that for the number of transmit antenna $\mathrm{N}_{\mathrm{t}}$, the number of input symbol that an encoder takes $(k)$ is equal to the number of time period $(p)$ required to transmit these symbols. For example, the transmission matrix $X_{4}$ for transmit antenna $N_{t}=4$, encoder takes the input symbol $x_{1}, x_{2}, x_{3}$, $x_{4}(k=4)$ and formulate the code sequence. At a time $t=1$ signal $x_{1}, x_{2}, x_{3}, x_{4}$ are transmitted from antenna 1 through 4. At a time $\mathrm{t}=2-x_{2}, x_{1},-x_{4}, x_{3}$ are transmitted from antenna 1 through 4 . At a time $t=3$ signal $-x_{3}, x_{4}$, $x 1,-x 2$ are transmitted from antenna 1 through 4 . So for four transmit antennas, four time periods are needed to transmit four message symbols. Hence it is obvious that this scheme requires no bandwidth expansion.

\subsection{Orthogonal Space-Time Block Codes}

The Alamouti scheme discussed in Section 4.3 is part of a general class of STBCs known as Orthogonal Space-Time Block Codes (OSTBCs) [4]. The authors of [15] apply the mathematical framework of orthogonal designs to construct both real and complex orthogonal codes that achieve full diversity. For the case of real orthogonal codes, it has been shown that a full rate code can be constructed [15]. However, for the case of complex orthogonal codes, it is unknown if a full rate and full diversity codes exist for $N_{t}>2$ [4]. Complex modulation techniques are of interest in this paper and therefore real orthogonal codes are not discussed. In next sections, the full diversity complex orthogonal codes presented in [15] for different rates are briefly introduced.

\subsection{1) Orthogonal Space-Time Block Codes for $N=3$ :}

For the case of 3 transmit antennas, Tarokh et al. construct block codes for the with $1 / 2$ and $3 / 4$ coding rate and full diversity $3 \mathrm{~N}_{\mathrm{r}}$.

a) $N_{t}=3$ with Rate $1 / 2$ : The full diversity, rate $1 / 2$ code for $N_{t}=3$ is given by [15], [11]:

$$
\left[\begin{array}{ccc}
\mathrm{s}_{1} & \mathrm{~s}_{2} & \mathrm{~s}_{3} \\
-\mathrm{s}_{2} & \mathrm{~s}_{1} & -\mathrm{s}_{4} \\
-\mathrm{s}_{3} & \mathrm{~s}_{4} & \mathrm{~s}_{1} \\
-\mathrm{s}_{4} & -\mathrm{s}_{3} & \mathrm{~s}_{2} \\
\mathrm{~s}_{1}^{*} & \mathrm{~s}_{2}^{*} & \mathrm{~s}_{3}^{*} \\
-\mathrm{s}_{2}^{*} & \mathrm{~s}_{1}^{*} & -\mathrm{s}_{4}^{*} \\
-\mathrm{s}_{3}^{*} & \mathrm{~s}_{4}^{*} & \mathrm{~s}_{1}^{*} \\
-\mathrm{s}_{4}^{*} & -\mathrm{s}_{3}^{*} & \mathrm{~s}_{2}^{*}
\end{array}\right]
$$

This code transmits 4 symbols every 8 time intervals, and therefore has rate $1 / 2$. The decision metric to minimize by the decoder for detecting s1, s2, s3, s4 are given by (10), (11), (12), (13) respectively where 


$$
\xi=\left(-1+2 \sum_{i=1}^{N_{r}} \sum_{j=1}^{N_{t}}\left|h_{i, j}\right|^{2}\right)
$$

for $\mathrm{N}_{\mathrm{t}}=3$.

$$
\begin{aligned}
& \left|\left[\sum_{i=1}^{N_{r}}\left(r_{i}^{(1)} h_{i, 1}^{*}+r_{i}^{(2)} h_{i, 2}^{*}+r_{i}^{(3)} h_{i, 3}^{*}+r_{i}^{(5)} h_{i, 1}^{*}+r_{i}^{(6)} h_{i, 2}^{*}+r_{i}^{(7)} h_{i, 3}^{*}\right)\right]-s_{1}\right|^{2}+\xi\left|s_{1}\right|^{2} \\
& \left|\left[\sum_{i=1}^{N_{r}}\left(r_{i}^{(1)} h_{i, 2}^{*}+r_{i}^{(2)} h_{i, 1}^{*}+r_{i}^{(4)} h_{i, 3}^{*}+r_{i}^{*(5)} h_{i, 2}-r_{i}^{*(6)} h_{i, 1}+r_{i}^{*(8)} h_{i, 3}\right)\right]-s_{2}\right|^{2}+\xi\left|s_{2}\right|^{2} \\
& \left|\left[\sum_{i=1}^{N_{r}}\left(r_{i}^{(1)} h_{i, 3}^{*}-r_{i}^{(3)} h_{i, 1}^{*}-r_{i}^{(4)} h_{i, 2}^{*}+r_{i}^{*(5)} h_{i, 3}-r_{i}^{*(7)} h_{i, 1}-r_{i}^{*(8)} h_{i, 2}\right)\right]-s_{3}\right|^{2}+\xi\left|s_{3}\right|^{2} \\
& \left|\left[\sum_{i=1}^{N_{r}}\left(-r_{i}^{(2)} h_{i, 3}^{*}+r_{i}^{(3)} h_{i, 2}^{*}-r_{i}^{(4)} h_{i, 1}^{*}-r_{i}^{*(6)} h_{i, 3}+r_{i}^{*(7)} h_{i, 2}-r_{i}^{*(8)} h_{i, 1}\right)\right]-s_{4}\right|^{2}+\xi\left|s_{4}\right|^{2}
\end{aligned}
$$

b) $N_{t}=3$ with Rate 3/4: A higher rate code with $N_{t}=3$ is given by [14], [15]:

$$
H_{3}=\left(\begin{array}{ccc}
s_{1} & s_{2} & \frac{s_{3}}{\sqrt{2}} \\
-s_{2}^{*} & s_{1}^{*} & \frac{s_{3}}{\sqrt{2}} \\
\frac{s_{3}^{*}}{\sqrt{2}} & \frac{s_{3}^{*}}{\sqrt{2}} & \frac{-s_{1}-s_{1}^{*}+s_{2}-s_{2}^{*}}{2} \\
\frac{s_{3}^{*}}{\sqrt{2}} & -\frac{s_{3}^{*}}{\sqrt{2}} & \frac{-s_{2}+s_{2}^{*}+s_{1}-s_{1}^{*}}{2}
\end{array}\right)
$$

As can be observed, (14) transmits 3 symbols every 4 time intervals, and therefore has rate $3 / 4$. The decision statistic to minimize for detecting $\mathrm{s}_{1}, \mathrm{~s}_{2}$, and $\mathrm{s}_{3}$ are given by (15), (16) and (17) respectively

$$
\begin{aligned}
& \left|\left[\sum_{i=1}^{N_{r}}\left(r_{i}^{(1)} h_{i, 1}^{*}+r_{i}^{(2)} h_{i, 2}^{*}+\frac{\left(r_{i}^{(4)}-r_{i}^{(3)}\right) h_{i, 3}^{*}}{2}-\frac{\left(r_{i}^{(3)}+r_{i}^{(4)}\right) h_{i, 3}^{*}}{2}\right)\right]-s_{1}\right|^{2}+\psi\left|s_{1}\right|^{2} \\
& \left|\left[\sum_{i=1}^{N_{r}}\left(r_{i}^{(1)} h_{i, 2}^{*}-r_{i}^{(2)} h_{i, 1}^{*}+\frac{\left(r_{i}^{(4)}+r_{i}^{(3)}\right) h_{i, 3}^{*}}{2}-\frac{\left(-r_{i}^{(3)}+r_{i}^{(4)}\right) h_{i, 3}^{*}}{2}\right)\right]-s_{2}\right|^{2}+\psi\left|s_{2}\right|^{2}
\end{aligned}
$$




$$
\left|\left[\sum_{i=1}^{N_{r}}\left(\frac{\left(r_{i}^{(1)}+r_{i}^{(2)}\right) h_{i, 3}^{*}}{\sqrt{2}}+\frac{r_{i}^{*(3)}\left(h_{i, 1}+h_{i, 2}\right)}{\sqrt{2}}+\frac{r_{i}^{*(4)}\left(h_{i, 1}-h_{i, 2}\right)}{\sqrt{2}}\right)\right]-s_{3}\right|^{2}+\psi\left|s_{3}\right|^{2}
$$

\subsection{2) Orthogonal Space-Time Block Codes for $\mathrm{Nt}=4$ :}

For the case of 4 transmit antennas, [15] provide block codes of rate $1 / 2$ and 3/4, both of which have full diversity $4 \mathrm{~N}_{\mathrm{r}}$.

a) $N_{t}=4$ with Rate $1 / 2$ : In the case of 4 transmit antennas, the rate $1 / 2$ code block is given by [11], [15]:

$$
\left(\begin{array}{cccc}
s_{1} & s_{2} & s_{3} & s_{4} \\
-s_{2} & s_{1} & -s_{4} & s_{3} \\
-s_{3} & s_{4} & s_{1} & -s_{2} \\
-s_{4} & -s_{3} & s_{2} & s_{1} \\
s_{1}^{*} & s_{2}^{*} & s_{3}^{*} & s_{4}^{*} \\
-s_{2}^{*} & s_{1}^{*} & -s_{4}^{*} & s_{3}^{*} \\
-s_{3}^{*} & s_{4}^{*} & s_{1}^{*} & -s_{2}^{*} \\
-s_{4}^{*} & -s_{3}^{*} & s_{2}^{*} & s_{1}^{*}
\end{array}\right)
$$

where, similar to (18), has rate $1 / 2$ as 4 symbols are transmitted in 8 time intervals. To decode, the ML decoder minimizes the decision metric (19), (20), (21), and (22) for decoding $\mathrm{s}_{1}, \mathrm{~s}_{2}, \mathrm{~s}_{3}$, and $\mathrm{s}_{4}$ respectively where is given by (19) for $\mathrm{N}_{\mathrm{t}}=4$. The decoding decision metric (22) for decoding $\mathrm{s}_{4}$ differs from that of [11] since the author discovered a mistake in the metric provided by Tarokh et al.

$$
\begin{aligned}
& \left|\left[\sum_{i=1}^{N_{r}}\left(r_{i}^{(1)} h_{i, 1}^{*}+r_{i}^{(2)} h_{i, 2}^{*}+r_{i}^{(3)} h_{i, 3}^{*}+r_{i}^{(4)} h_{i, 4}^{*}+r_{i}^{(5)} h_{i, 1}+r_{i}^{(6)} h_{i, 2}+r_{i}^{(7)} h_{i, 3}+r_{i}^{(8)} h_{i, 4}\right)\right]-s_{1}\right|^{2}+\xi\left|s_{1}\right|^{2} \\
& \left|\left[\sum_{i=1}^{N_{r}}\left(r_{i}^{(1)} h_{i, 2}^{*}-r_{i}^{(2)} h_{i, 1}^{*}-r_{i}^{(3)} h_{i, 4}^{*}+r_{i}^{(4)} h_{i, 3}^{*}+r_{i}^{*(5)} h_{i, 2}-r_{i}^{*(6)} h_{i, 1}-r_{i}^{*(7)} h_{i, 4}+r_{i}^{*(8)} h_{i, 3}\right)\right]-s_{2}\right|^{2}+\xi\left|s_{2}\right|^{2} \\
& \left|\left[\sum_{i=1}^{N_{r}}\left(r_{i}^{(1)} h_{i, 3}^{*}+r_{i}^{(2)} h_{i, 4}^{*}-r_{i}^{(3)} h_{i, 4}^{*}-r_{i}^{(4)} h_{i, 2}^{*}+r_{i}^{*(5)} h_{i, 3}+r_{i}^{*(6)} h_{i, 4}-r_{i}^{*(7)} h_{i, 1}-r_{i}^{*(8)} h_{i, 2}\right)\right]-s_{3}\right|^{2}+\xi\left|s_{3}\right|^{2} \\
& \left|\left[\sum_{i=1}^{N_{r}}\left(r_{i}^{(1)} h_{i, 4}^{*}-r_{i}^{(2)} h_{i, 3}^{*}+r_{i}^{(3)} h_{i, 2}^{*}-r_{i}^{(4)} h_{i, 4}^{*}+r_{i}^{*(5)} h_{i, 4}-r_{i}^{*(6)} h_{i, 3}+r_{i}^{*(7)} h_{i, 2}-r_{i}^{*(8)} h_{i, 1}\right)\right]-s_{4}\right|^{2}+\xi\left|s_{4}\right|^{2}
\end{aligned}
$$




\section{Adaptation Modulation and Coding (AMC) Scheme}

In reaction to an observed packet error rate, $\mathrm{p}_{\mathrm{cur}}$, in order to insure packet errors correction, we propose to apply instantaneously an adaptive FEC mechanism based on MDS codes, of which the FEC ratio is a function of the current packet error rate, $\mathrm{f}_{\text {cur }}=\mathrm{g}\left(\mathrm{p}_{\text {cur }}\right)$ to Network SDUs. If we consider that a group of Network-SDU includes w Network-SDUs, then the number of redundancy packets (with the same packet size of 1024 bytes) for each group is given by:

$$
m=\text { ceiling }\left(w * \frac{f_{\text {cur }}}{1-f_{\text {cur }}}\right)
$$

The FEC ratio $f_{\text {cur }}=g\left(p_{\text {cur }}\right)=p_{\text {cur }}$ is in a first step based on the hypothesis of an uniform packet error distribution that makes possible the full recovery of the lost packets. Note that this hypothesis is reasonable hen the FEC ratio processing is applied to short periods that make it possible an adaptation to non uniform packet error distributions. In the context of UDP based transmission, within time slots $\mathrm{t}$ and subcarriers $\mathrm{s}$ assigned by $\mathrm{BS}$, we denote respectively $R_{c}$ : the current UL transmission rate of a mobile node, $R_{l}$ : the UL transmission rate if the mobile node uses the next lower modulation/coding scheme, $R_{h}$ : the UL transmission rate if the mobile node uses the next higher modulation/coding scheme. Then, the UL throughput within the assigned time slots (useful transmission rate successfully received by the BS during t) is given by:

$$
G_{c}=R_{c} *\left(1-f_{\text {cur }}\right)=R_{c} *\left(1-p_{\text {cur }}\right)
$$

The object of our contribution is to choose an optimal combination of the high layer FEC and the default adaptive modulation and coding AMC scheme. Similar to the previous analysis, if we set the FEC ratio $f_{i}=p_{i}$ $\mathrm{SNR}$, then the UL throughput becomes $G_{i}$ if the mobile node uses the neighbour modulations/coding schemes:

$$
G_{i}=R_{i} *\left(1-f_{i}\right)=R_{i} *\left(1-p_{S N R}^{i}\right) \quad \text { with } i=h \text { or } i=l
$$

The spectral efficiency $\mathrm{C}$ is denned as:

$$
C=\text { coderate } * \log _{2}^{(M)}
$$

where code rate and $M$ represent respectively the coding rate and the $M$-ary phase.

\section{Experimental Results}

A real time software defined radio (SDR) is developed (as shown in Fig. 3) by using a laptop with 8 Giga Bytes of RAM and an Intel ${ }^{\circledR}$ Core ${ }^{\mathrm{TM}}$ i5-3210M CPU clocked at $2.50 \mathrm{GHz}$. The integrated 1000Base-T Ethernet interface was connected to the USRPN210, equipped with the CBX daughterboard which is a fullduplex, wide band transceiver that extends a frequency band from $1.2 \mathrm{GHz}$ to $6 \mathrm{GHz}$ with a instantaneous bandwidth of $40 \mathrm{MHz}$. As indicated in Fig. 3, the incoming signal from the file source is channel coded by the scrambler, RMG encoder and interleaving separately and passed through the OFDM modulator to produce OFDM symbols and afterwards passed through the multiply constant block and channel model. The multiply constant block is utilized to enhance the amplitude of the OFDM symbols. The channel model could be reconfigured by changing frequency offset, noise parameters. The generated OFDM symbols are demodulated, deinterleaved, decoded and descrambled respectively. The concatenated OFDM signal is transmitted by USRP 
N210 RF front end by using TX/RX antenna and received by RX2 antenna over air (see Fig. 3) in the lab environment. The RMG coder is replaced with Convolutional coder, RM coder and results are noted in Table 1 by varying modulation schemes and bits per byte in BER block. It can be concluded that BER performance is improved as number of bits per symbol is increased and varies with window size. As modulation scheme size increases BER also increased (Observe Table 1) which is not desirable. Hence, while preferring a type of modulation scheme, various parameters have to be taken in to consideration.

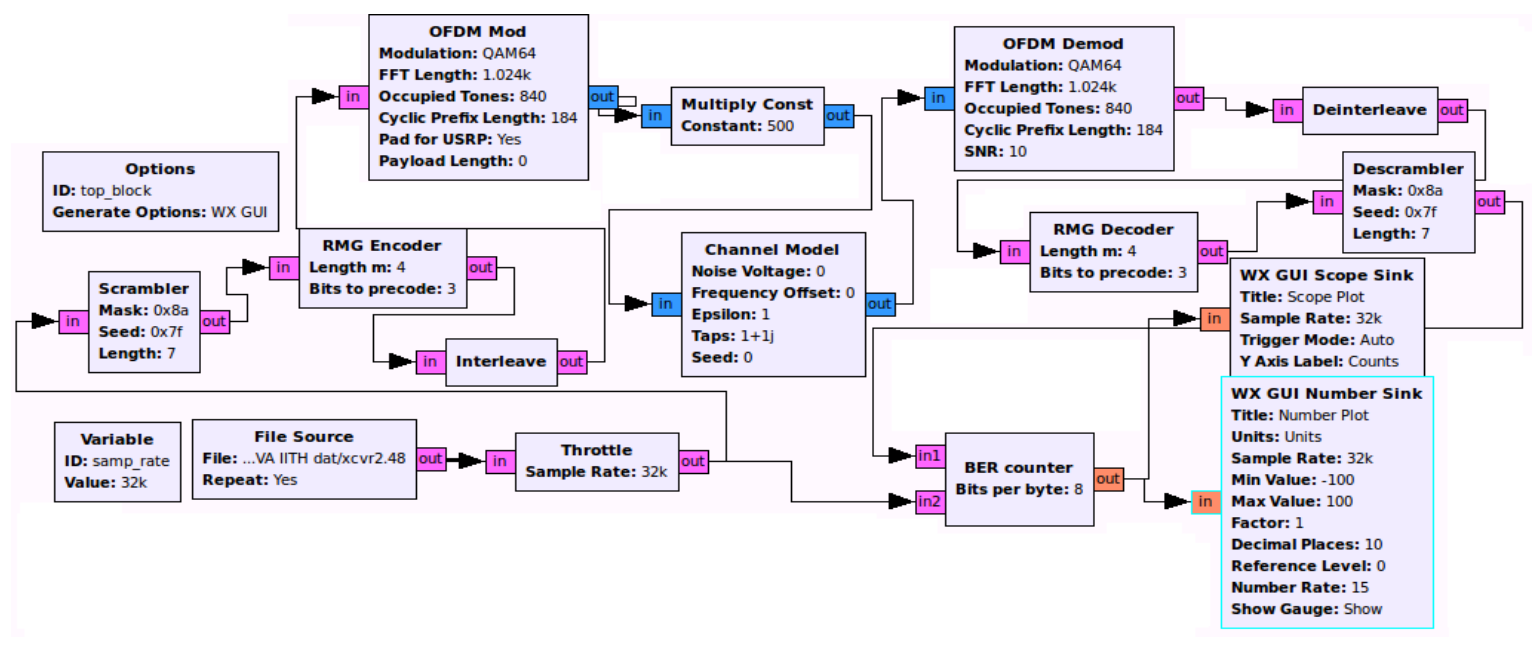

Fig. 3. GNU Schematic for BER of 64QAM-OFDM with RMG coder channel coding.

Table 1. Results Obtained For BER Performance with Channel Coding Over AWGN Channel.

\begin{tabular}{|l|l|l|l|}
\hline Coder & Mod Scheme & Bits per byte & BER value \\
\hline RMG Coder & BPSK & 1 & 0.5001434684 \\
\hline RMG Coder & BPSK & 4 & 0.4068774879 \\
\hline RMG Coder & BPSK & 8 & 0.3892548680 \\
\hline & & Average & 0.43209194 \\
\hline RMG Coder & QAM64 & 1 & 0.5004814267 \\
\hline RMG Coder & QAM64 & 4 & 0.4189103246 \\
\hline RMG Coder & QAM64 & 8 & 0.4092760682 \\
\hline & & Average & 0.44288927 \\
\hline CCSDS Coder & BPSK & 1 & 0.4585958719 \\
\hline CCSDS Coder & BPSK & 4 & 0.5740551949 \\
\hline CCSDS Coder & BPSK & 8 & 0.5869382620 \\
\hline & & Average & 0.53986311 \\
\hline CCSDS Coder & QAM64 & 1 & 0.4601847827 \\
\hline CCSDS Coder & QAM64 & 4 & 0.5721712112 \\
\hline CCSDS Coder & QAM64 & 8 & 0.5870822072 \\
\hline & & Average & 0.53981274 \\
\hline RM Coder & BPSK & 1 & 0.5000710487 \\
\hline RM Coder & BPSK & 4 & 0.4210021496 \\
\hline RM Coder & BPSK & 8 & 0.3852755427 \\
\hline & & Average & 0.43544958 \\
\hline RM Coder & QAM64 & 1 & 0.5001475811 \\
\hline RM Coder & QAM64 & 4 & 0.42152449419 \\
\hline RM Coder & QAM64 & 8 & 0.4092907906 \\
\hline & & Average & 0.44365444 \\
\hline
\end{tabular}


The OSTBC performance for the case of $N_{r}=1$ is shown in Fig. 4. As expected, for each different code blocks, the performance degrades as more bits per symbol are transmitted. It can be observed that for a particular modulation and high SNR, the best performance is obtained by G4 followed by $H_{4}, G_{3}, H_{3}$, and $G_{2}$. However, for any modulation and low SNR, $G_{3}$ out performs $H_{4}$ even when $H_{4}$ has great gain. The results is that the best performance at low SNR is obtained by $\mathrm{G}_{4}$ followed by $G_{3}, H_{4}, H_{3}$, and $G_{2}$. Moreover $H_{4}$ is outperformed by $G_{3}$ for the cases of $N_{r}=2, N_{r}=3$ and $N_{r}=4$.

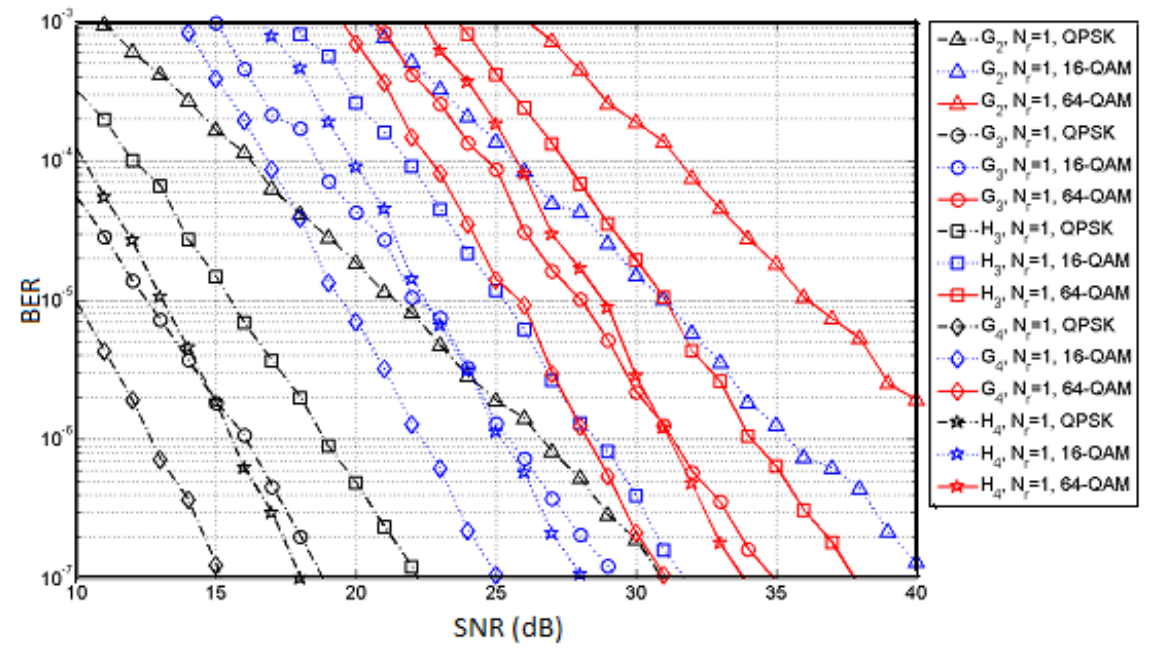

Fig. 4. BER of OSTBC performance for $\mathrm{Nr}=1$ in Rayleigh channel.

The OSTBC performance for the case of $\mathrm{N}_{\mathrm{r}}=4$ is shown in Fig. 5. As can be observed, for a particular modulation, the best performance is obtained by G4 followed by G3, H4, H3, and G2. This order is the same as for the case of $\mathrm{Nr}=1$ and low SNR where G3 outperforms H4 even with $\mathrm{H} 4$ having higher gain. One possible reason for this behaviour is that the higher rate of $\mathrm{H} 4$ causes lower channel gain per symbol and therefore higher BER for a particular SNR.

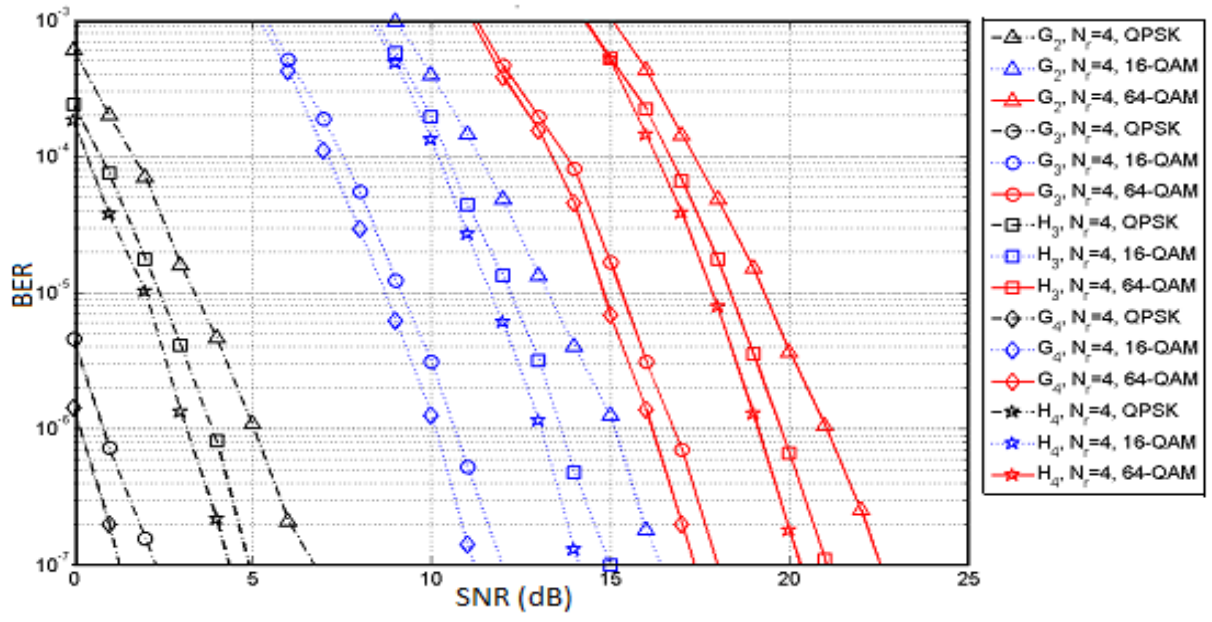

Fig. 5. BER of OSTBC performance for $\mathrm{Nr}=4$ in Rayleigh channel. 
The BER curve for the case of keeping $N_{t}=4$ constant while varying $N_{r}$ from 1 to 4 for different modulations is depicted in Fig. 6. It can be observed that for any modulation and block code, the gain of using 3 more antennas is approximately $14 \mathrm{~dB}$. However, between $N_{r}=1$ and $N_{r}=2$ the gain is approximately $8 \mathrm{~dB}$, between $N_{r}=2$ and $N_{r}=3$ the gain is approximately $4 \mathrm{~dB}$, and between $N_{r}=3$ and $N_{r}=4$ the gain is approximately $2 \mathrm{~dB}$. This result suggest diminishing returns as $N_{r}$ increases. Another observation is that for any $\mathrm{N}_{\mathrm{r}}$ and modulation scheme, $G_{3}$ and $G_{4}$ have a $3 \mathrm{~dB}$ gain over $H_{3}$ and $H_{4}$ respectively. An interesting observation is that the performance of $G_{4}$ with $N_{r}=2$ is similar to that of $\mathrm{H}_{4}$ with $N_{r}=3$, while $\mathrm{G}_{4}$ with $N_{r}=1$ is outperformed by $H_{4}$ with $N_{r}=2$, and $G_{4}$ with $N_{r}=3$ outperforms $\mathrm{H}_{4}$ with $N_{r}=4$.

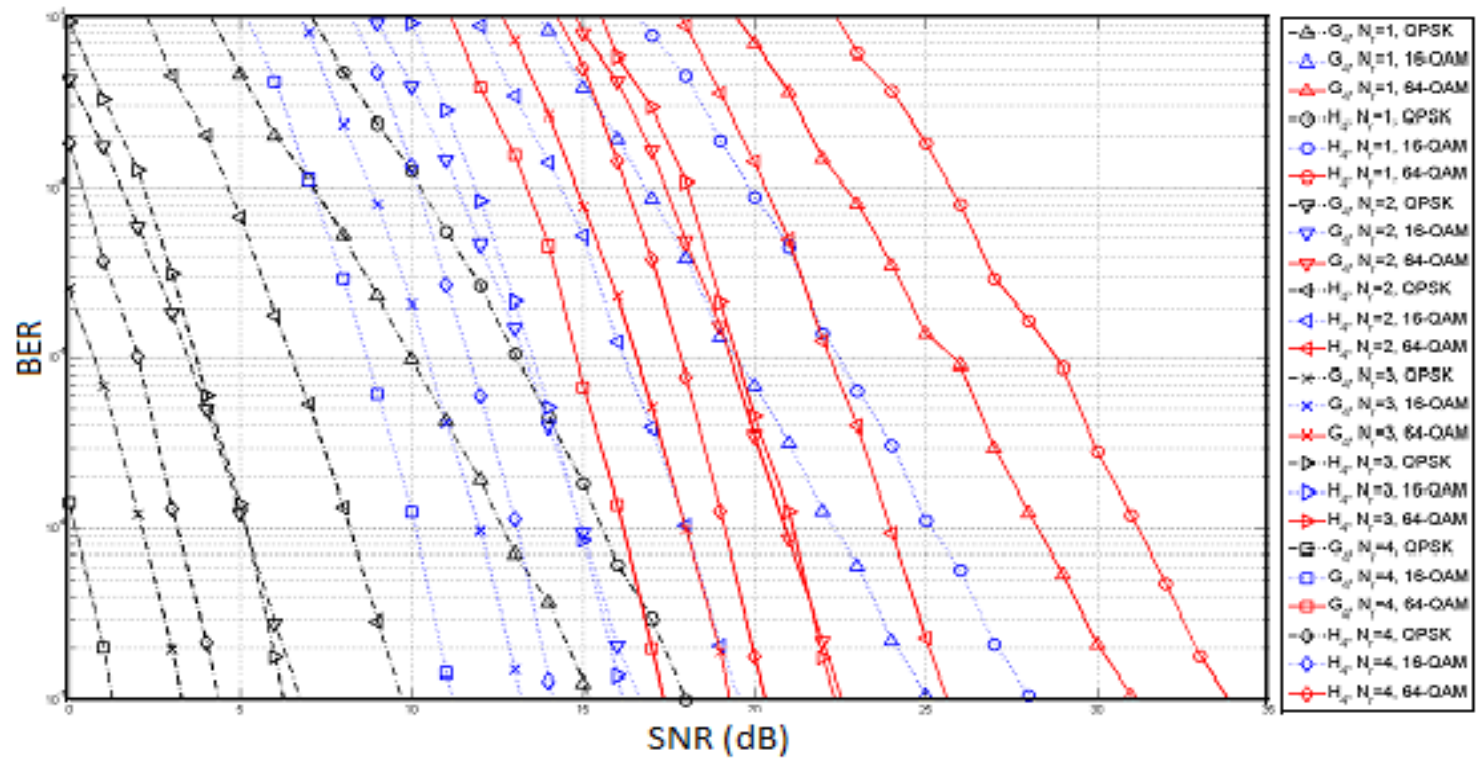

Fig. 6. BER of OSTBC for $\mathrm{Nt}=4$ in Ralyleigh channel.

Fig. 7 represents the default and the FEC based throughput experienced by the mobile node. Fig. 8 represents the gain of UL throughput with our proposal compared to the default method. Fig. 9 represents the FEC ratio at higher layer according to the SNR value. We observe that the throughput gain is not homogeneously spatially distributed along the radius of the WiMAX coverage. In the following, we will estimate the percentage of the WiMAX coverage, approximated by a disk, where a gain can be observed. In order to estimate this ratio, we will use the model introduced in [14] for the processing of the distance between the BS and a MN in open air (D) according to the monitored SNR:

$$
\begin{aligned}
& E=\frac{P E[d B m]+10 \log (G E) d B+10 \log [(G R)(d B)]-S N R[d B]-N[d B m]}{20} \\
& D=\frac{\lambda * 10 \exp (E)}{4 * \pi}
\end{aligned}
$$

Where PE is the emitted power, GE is the emitter antenna gain, GR is the receiver antenna gain, $\mathrm{N}$ is the thermal noise and $\lambda$ is the wavelength. If we assume a frequency of $3.4 \mathrm{GHz}$ used for the outdoor WiMAX in France and the thermal noise is equal to $-100.97 \mathrm{dBm}$. 


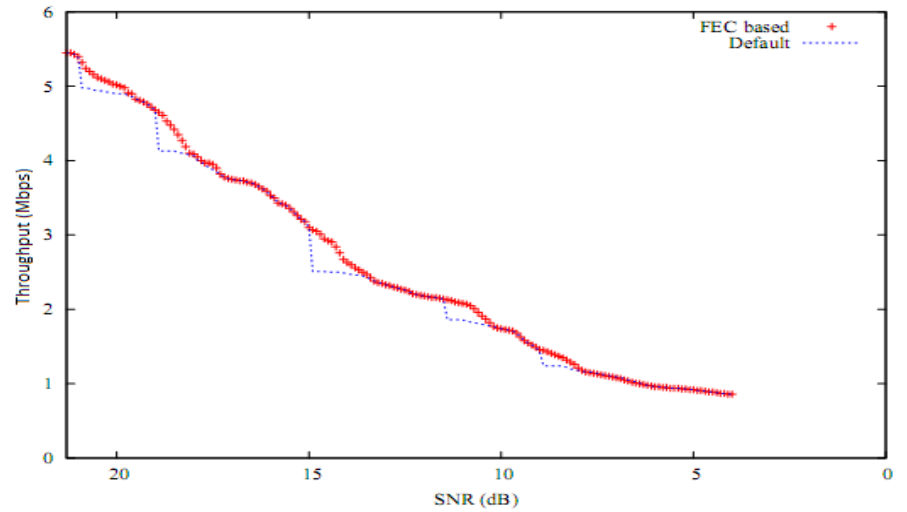

Fig. 7. Default and FEC based UL throughput.

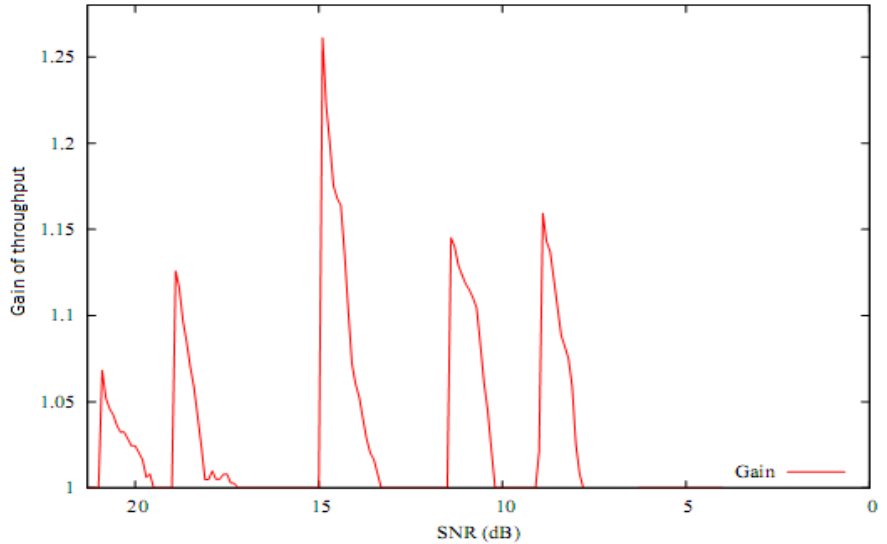

Fig. 8. Gain of UL throughput.

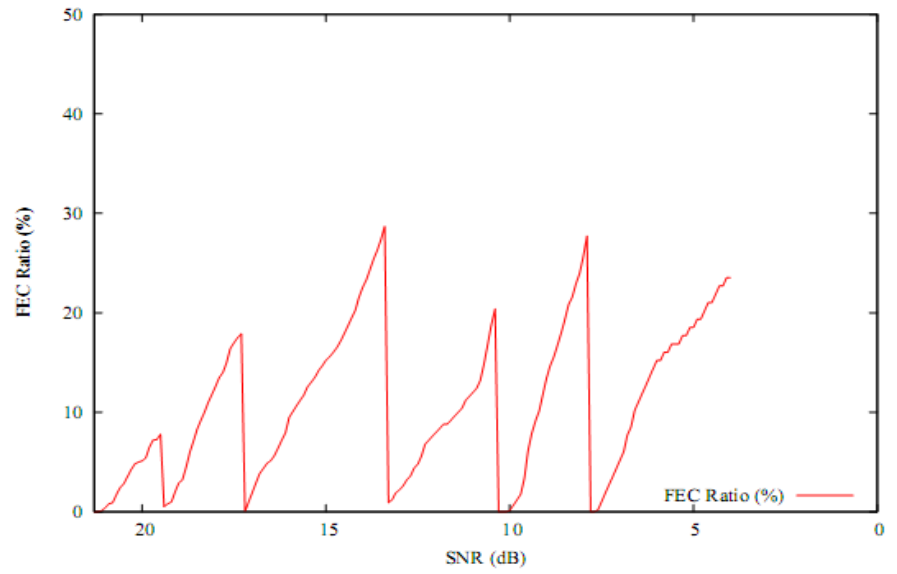

Fig. 9. FEC ratio according to SNR. 
The simulation results show that our proposal always offers a higher UL throughput compared to the default method. Since the FEC mechanism is implemented in the high layer, our proposal does not induce modifying the WiMax standard but simply requires a simple thin interface between the MAC and network layer for the management of the mapping database and high layer adaptive erasure code. This practical consideration makes our proposal compliant with a fast deployment.

\section{Conclusion}

This paper has demonstrated the implementation of WiMAX PHY with adaptive modulation techniques using software defined radio (SDR) testbed. For the development of SDR, GNU Radio and USRP N210 are employed as software and hardware platforms respectively. It was observed that higher diversity gain does not always imply better performance. This was observed when $\mathrm{G}_{3}$ outperformed $\mathrm{H}_{4}$ at low $\mathrm{SNR}$ for $\mathrm{Nr}=1$ and at any SNR for $\mathrm{Nr}=2$ up to $\mathrm{Nr}=4$. Similarly, it was observed that equal diversity gain does not imply equal performance. This was particularly demonstrated when $\mathrm{G}_{3}$ outperformed all others for equal diversity gain. The penalty of having more transmit antennas, which consequently reduces the energy per transmit antenna was observed. Also, we observed diminishing returns for every scheme as the number of received antennas increased. The analytical study and the simulation results introduced in this paper demonstrate a significant improvement on the throughput and transmission efficiency offered to WiMAX mobile nodes.

\section{References}

[1] Shi, Wang, Wang Yingchun, and Guan Jianxin. "A New Ranging Technique for IEEE 802.16 e Uplink." International Journal of Wireless and Microwave Technologies (IJWMT), vol. 1, no. 4, 2011.

[2] Pan, Yuwen, Andrew Nix, and Mark Beach, "Distributed resource allocation for OFDMA-based relay networks", IEEE Transactions on Vehicular Technology, vol. 60, no. 3, pp. 919-931, 2011.

[3] Sur, Samarendra Nath, and Debjyoti Ghosh. "Channel Capacity and BER Performance Analysis of MIMO System with Linear Receiver in Nakagami Channel." International Journal of Wireless and Microwave Technologies (IJWMT), vol. 3, no. 1, 2013.

[4] Nee, Richard van and Prasad, Ramjee, "OFDM for wirelessmultimedia communications", Artech House, 2000.

[5] Rabiei, Payam, Won Namgoong, and Naofal Al-Dhahir, "A non-iterative technique for phase noise ICI mitigation in packet-based OFDM systems", IEEE Transactions on Signal Processing, vol. 58, no. 11, pp. 5945-5950, 2010.

[6] K. Zheng, L. Huang, W. Wang, and G. Yang, "TD-CDM-OFDM: Evolution of TD-SCDMA toward 4G," IEEE Communications Magazine, vol. 43, no. 1, pp. 45-52, 2005.

[7] H. Sampath, S. Talwar, J. Tellado, V. Erceg, and A. Paulraj, "A fourth-generation MIMO-OFDM broadband wireless system: design, performance, and field trial results," IEEE Communications Magazine, vol. 40, no. 9, pp. 143-149, 2002.

[8] Kildal, P-S., et al., "Threshold receiver model for throughput of wireless devices with MIMO and frequency diversity measured in reverberation chamber," IEEE Antennas and Wireless Propagation Letters, vol. 10, pp. 1201-1204, 2011.

[9] Xiong, Cong, et al., "Energy-and spectral-efficiency tradeoff in downlink OFDMA networks", IEEE Transactions on Wireless Communications, vol. 10, no.11, pp. 3874-3886, 2011.

[10] Chae, Chan-Byoung, et al., "Adaptive MIMO transmission techniques for broadband wireless communication systems [Topics in Wireless Communications]", IEEE Communications Magazine, vol. 48, no. 5, pp. 112-118, 2010.

[11] Shen, Godwin, et al., "Edge-adaptive transforms for efficient depth map coding", IEEE Picture Coding Symposium (PCS), 2010.

[12] Lei Zhang, Patrick S'enac, Roksana Boreli and Michel Diaz, "Optimization of WiMax modulation scheme with a 
cross layer erasure code", 2008.

[13] Cortes-Pena, Luis Miguel. "MIMO Space-Time Block Coding (STBC): Simulations and Results." Design Project: Personal And Mobile Communications, Georgia Tech (ECE6604), 2009.

[14] V. Tarokh, H. Jafarkhani, and A. Calderbank, "Space-time block coding for wireless communications: performance results," IEEE Journal on selected areas in communications, vol. 17, no. 3, pp. 451-460, 1999.

[15] V. Tarokh, H. Jafarkhani, and A. Calderbank, "Space-time block codes from orthogonal designs," IEEE Transactions on Information Theory, vol. 45, no. 5, pp. 1456-1467, 1999.

\section{Authors' Profiles}

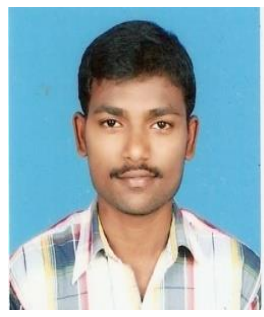

B. Siva Kumar Reddy, has received B.Tech (E.C.E) and M.Tech (VLSI Design (Very Large Scale Integrated circuits Design)) degrees from Jawaharlal Nehru Technological University, Hyderabad (JNTUH), India. Currently, he is working for doctorate in the field of wireless communications at National Institute of Technology Warangal, India. He is an IEEE student member, ISTE (Indian Society for Technical Education) life time member and IDES (Institute of Doctors, Engineers and Scientists) member. Email: bsivakumar100@gmail.com

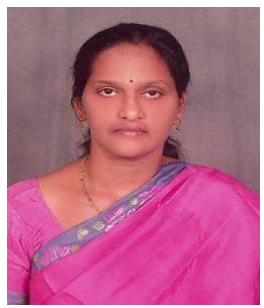

Dr. B. Lakshmi, has obtained B.Tech (E.C.E) from Nagarjuana university, M.Tech (EI) from NIT, Warangal, and Ph.D (VLSI Architectures) from I.I.T, Kharagpur, India. She is working as a faculty member in National Institute of Technology, Warangal since 1990. Her areas of interests are Digital System Design, Microprocessor Systems and VLSI Architectures. She is reviewer for Elsevier journals in VLSI area of Technology.

How to cite this paper: B. Siva Kumar Reddy, B. Lakshmi,"BER Analysis with Adaptive Modulation Coding in MIMO-OFDM for WiMAX using GNU Radio", IJWMT, vol.4, no.4, pp.20-34, 2014.DOI: 10.5815/ijwmt.2014.04.02 